\title{
Long-Term Trajectories of BMI Predict Carotid Stiffness and Plaque Volume in Type 2 Diabetes Older Adults: a Cohort Study.
}

chen Botvin Moshe ( $\square$ chen.botvin@gmail.com)

Tel Aviv University https://orcid.org/0000-0002-4894-9659

Salo Haratz

Neurology Department, Assuta Ashdod Hospital

Ramit Ravona-Springer

Sheba Medical Center at Tel Hashomer

Anthony Heymann

Maccabi health Services, Tel Aviv University Sackler Faculty of Medicine

\section{Lin Hung-Mo}

Icahn School of Medicine at Mount Sinai

Michal Schnaider Beeri

The Josef Sagol Neuroscience Center, Sheba Medical Center, Mount Sinai Beth Israel Hospital Department of Psychiatry

David Tanne

Rambam Health Care Campus, Stroke and cognition institute

Original investigation

Keywords: Obesity, Diabetes, Carotid Atherosclerosis.

Posted Date: August 6th, 2020

DOI: https://doi.org/10.21203/rs.3.rs-20601/v2

License: (c) (i) This work is licensed under a Creative Commons Attribution 4.0 International License. Read Full License

Version of Record: A version of this preprint was published on September 15th, 2020. See the published version at https://doi.org/10.1186/s12933-020-011046. 


\section{Abstract}

Background: High body mass index (BMI) is a risk factor for type 2 diabetes and cardiovascular disease. However, its relationships with indices of carotid stiffness and plaque volume are unclear. We investigated associations of long-term measurements of BMI with indices of carotid stiffness and atherosclerosis among non-demented diabetes patients from the Israel Diabetes and Cognitive Decline (IDCD) study.

Methods: Carotid ultrasound indices [carotid intima media thickness (cIMT), distensibility, elastography and plaque volume] were assessed in $\mathrm{N}=471$ participants. Mean BMI across all MHS diabetes registry measurements and trajectories of BMI were calculated. BMI was categorized into three trajectory groups representing: a relatively stable normal weight $(n=185,44 \%)$, overweight trajectory $(n=188,44.8 \%)$ and a trajectory of obesity $(n=47,11.2 \%)$. Linear and logistic regressions estimated associations of carotid indices with mean BMI and BMI trajectories.

Results: Compared to the normal weight trajectory, an obesity trajectory was associated with carotid distensibility $(\beta=-3.078, p=0.037)$, clMT $(\beta=0.095$, $p=0.004)$, and carotid elastography $(\beta=0.181, p=0.004)$ but not with plaque volume $(\beta=0.066, p=0.858)$. Compared with the normal weight trajectory, an obesity trajectory was associated with increased odds for impaired carotid distensibility $(O R=2.790, p=0.033)$, impaired cIMT (OR=5.277, $p=0.001)$ and large carotid plaque volume $(\mathrm{OR}=8.456, \mathrm{p}=0.013)$ but not with carotid elastography $(\mathrm{OR}=1.956, \mathrm{p}=0.140)$. Mean $\mathrm{BMI}$ was linearly associated with Distensibility $(\beta=-0.275, p=0.005)$ and $\mathrm{cIMT}(\beta=0.005, \mathrm{p}=0.026)$.

Conclusions: Long-term measurements of adiposity are associated with indices of carotid stiffness and plaque volume among older type 2 diabetes adults.

\section{Background}

Overweight and obesity, commonly measured by body mass index (BMI) ${ }^{1,2}$ have been shown to be an independent risk factor for type 2 diabetes, hypertension, cardiovascular disease and stroke $e^{1}$. In the United States nearly $35 \%$ of the adults are obese and obesity is the $5^{\text {th }}$ leading cause of death ${ }^{3}$. The association of obesity with carotid atherosclerosis, as measured by cIMT and distensibility was demonstrated in several studies ${ }^{4-7}$. Vascular changes already develop among obese young children ${ }^{8}$ and adolescents ${ }^{9}$ suggesting that the exposure of obesity may affect vascular health throughout the life course.

Atherosclerosis is the underlying process of most cardiovascular disease. Carotid Intima Media Thickness (cIMT) was found to be a predictor for cardiovascular disease risk ${ }^{4,10}$, stroke ${ }^{11}$, and all-cause mortality ${ }^{12}$. Recently some innovative ultrasound methods have been developed to assess pre-clinical markers of carotid atherosclerosis disease and to evaluate its progression: carotid artery distensibility and carotid shear-wave elastography. Carotid distensibility is a functional parameter that measures the arterial ability to expand and contract with cardiac pulsation and relaxation ${ }^{13}$. Functional impairment of the arterial wall may occur at an early stage of the atherosclerotic process before structural wall changes become detectable as well as before the occurrence of clinical symptoms of vascular disease ${ }^{14}$. Shear wave elastography of the carotid wall is an innovative method used to evaluate carotid artery wall stiffness. Elastography measures the structural property of the carotid artery wall and represents the artery tissue stiffness.

Despite the key role of obesity in the incidence of type 2 diabetes, and the consistently increased risk for carotid atherosclerosis in type 2 diabetes patients, evidence on the associations of long-term obesity with indices of carotid stiffness and atherosclerotic plaque volume in this high-risk population are scarce. In this study, we used data from the Israel Diabetes and Cognitive Decline (IDCD) study to investigate the association of long-term measurements of BMI with ultrasound indices of carotid arterial wall function and atherosclerotic plaque volume in cognitively normal type 2 diabetes older adults.

\section{Methods}

\section{Study population}

The IDCD study investigates the effects of long-term type 2 diabetes-related characteristics on cognitive decline and the study design has been previously described in detail ${ }^{15}$. Briefly, the IDCD recruited community-dwelling elderly individuals with type 2 diabetes (65+ years old) living in central Israel, from approximately 11,000 clients enrolled in the diabetes registry of the Maccabi Healthcare Services (MHS). MHS is the second largest health maintenance organization (HMO), treating a representative cross-section of 2 million citizens. The MHS diabetes registry was established in 1998 to facilitate diabetes management and to improve treatment to include patients according to criteria previously detailed ${ }^{15}$. IDCD inclusion criteria were having type 2 diabetes; normal cognition at entry; being free of any neurological (e.g., Parkinson's disease, stroke), psychiatric (e.g., schizophrenia) or other diseases (e.g., alcohol or drug abuse) that might affect cognition; and having an informant. The IDCD cohort was established for the investigation of the contribution of T2D characteristics to cognitive decline among patients with type 2 diabetes. Therefore, the eligibility criteria were focused on factors that may be related to cognition rather than to global health. From the T2D angle, the goal was to include the broadest sample possible in terms of micro and macro complications, as those may themselves contribute to greater cognitive decline and dementia, so ketoacidosis, major hypoglycemia episodes, renal failure etc, were not exclusion criteria. Participants were assessed by a physician experienced in assessment and diagnosis of dementia, and by a neuropsychologist, who administered a broad neuropsychological battery. Four hundred and seventy-one (471) IDCD participants went through the carotid artery US assessments and had complete data on sociodemographic (Figure 1). The study was approved by the Institutional Review Boards of all three institutions participating in the study (Ichan School of Medicine at Mount Sinai, NY, Sheba Medical Center, Israel and Maccabi Health Services, Israel) and all participants signed informed consent.

\section{Ultrasound assessment procedures}

Carotid stiffness and atherosclerosis was assessed following the IDCD 36 months follow up visit. All examinations were performed at the Department of Neurology, Sheba Medical Center by one of 2 qualified and experienced ultrasound technicians, after obtaining informed consent. Subjects were placed in a 
supine position and rested for 5 minutes prior to assessing their vital signs. Carotid Ultrasound Doppler was performed using the premium EPIQ 7 US system (Philips, Netherlands). The following indices of carotid stiffness and atherosclerosis were assessed:

Carotid intima-media thickness (cIMT): IMT is defined as the distance between the media-adventitia interface and the lumen-intima interface. Measurements were performed bilaterally at the far wall of the common carotid artery (CCA) $1.0 \mathrm{~cm}$ proximal to the carotid bifurcation. The measurement was for a length of $10 \mathrm{~mm}$, which consisted of 150 points of measurements, on the far wall of the common carotid artery on both sides, as described in previous studies ${ }^{16}$. Plaque was defined according to the Mannheim consensus ${ }^{17}$, in which a plaque was diagnosed when the vessel wall thickness was $>1.5 \mathrm{~mm}$ or when the vessel wall appeared to be $\geq 0.5 \mathrm{~mm}$ or $50 \%$ thicker than the surrounding wall. The mean value of computer-based points was used. For each individual, clMT was determined as the average of 3 measurements for each artery, as was automatically computed by the QLAB software (Philips, Netherlands).

3-D Carotid plaque volume: Patients with detectable plaques in the carotid artery went through plaque volume analysis. In standard optimized mode, using the mechanic volumetric VL13-5 broadband linear array transducer, 3D plaque scanning volume data were obtained automatically. For each volume approximately 250 single transverse images (frames) were obtained with an interval of $0.15 \mathrm{~mm}$. Plaque volume was automatically calculated using the Vascular Plaque Quantification (VPQ) module (QLAB software), after selecting the beginning and ending frame and selecting at least one key frame within the plaque region.

Carotid Distensibility: Following static B-mode real-time imaging from a longitudinal section of the CCA after 5 minutes of rest, dynamic CINE looped M-mode images of consecutive cardiac cycles were stored for later offline analysis. Distensibility was assessed using the distension of both CCAs, measuring the change in diameter in systole relative to diastolic during the cardiac cycle. The vessel lumen diameter was assessed from the near wall to the far wall of the CCA. The maximal systolic lumen diameter was determined visually and from the R-wave of the ECG-recording and the minimal lumen diameter was used for the diastolic diameter. The end-diastolic diameter $(\mathrm{Dd})$, the absolute stroke change in diameter during systole $(\Delta \mathrm{A})$, and the relative stroke change in diameter $(\Delta \mathrm{A} / \mathrm{Dd})$ were computed as the mean of 10 cardiac cycles of one successive recording. Blood pressure was measured before and after the measurement session and pulse pressure $(\Delta \mathrm{P})$ was defined as the difference between the systolic and diastolic blood pressure. The cross-sectional arterial wall distensibility coefficient was calculated according to the following equation:

Cross sectional distensibility coefficient: (see Equation 1 in the Supplementary Files)

Carotid Elastography: In the B-mode display, a midsection of straight CCA in longitudinal plane is chosen. The shear wave elastographic mode was activated to show paired images of B-mode and elastography at the same time. The probe was handed with standardized pressure in the $2^{\text {nd }}-4^{\text {th }}$ quintile of the linear pressure scale, as seen using standardized real-time measurement displayed on a linear scale. Elastographic images are displayed with different color mapping for the softest, intermediate and hardest components, according to the different levels of strain. On a representative static image, the relative strain ratio (SR), between blood to carotid arterial wall were measured. The first region of interest (ROI) for the arterial wall strain was manually placed at the midpoint of posterior wall of displayed carotid artery. The second ROI for the blood strain was placed at the center of arterial lumen. SR was calculated automatically by dividing strain value of the blood by that of carotid arterial wall, using the QLAB software. Measurement were performed during 10 heart beats and an average of 3 images as described above, preferably consecutive, were used as the elastography index.

\section{Covariates}

Data on risk factors and possible confounders were obtained using the Maccabi Diabetes Registry and data collection during the baseline visit of the IDCD cohort. Variables available through the Maccabi Diabetes Registry were computed as the average of all the measurements done in Maccabi since the subject entered the Diabetes Registry. Variables extracted from Maccabi Diabetes Registry included time in the diabetes registry (a proxy of duration of diabetes) ${ }^{18}$, $\mathrm{BMI}, \mathrm{HbA1c}$ total, HDL and LDL cholesterol, triglycerides, CRP, eGFR, diabetes treatment and smoking. All blood measurements obtained from Maccabi were analyzed at a central lab. BMI was calculated using height and weight measured at the physician office. During the baseline visit of the IDCD study blood samples were obtained to evaluate CRP. Blood pressure was measured during the carotid artery US examination.

$H b A 1 c$

$\mathrm{HbA1c}$ was measured with standard methods of high-performance liquid chromatography using an ion exchange column. Participants were assessed under fasting conditions approximately annually at the MHS

CRP

C-reactive protein (mg/L) was measured from plasma, using the ADVIA 1650 Chemistry System with a CRP latex reagent.

\section{Statistical methods}

All variables were reviewed for abnormal values, to assess skewedness and outliers. Characteristics of study participants between the 3 trajectory groups were compared using independent-samples T-test, Wilcoxon rank-test and ANOVA, as appropriate for continuous variables, and $\chi^{2}$ test for categorical variables.

The outcomes (cIMT, distensibility coefficient and elastography strain ratio) were defined as the average of the measurements in the right and the left CCA. Carotid plaque volume total burden was defined as the sum of the plaque volume in the right and the left. Carotid plaque was categorized into 4 groups: no plaque group, and tertiles of the plaque volume: small plaque (volume $\leq 122 \mathrm{~mm}^{3}$ ) medium plaque (volume $122.1-271 \mathrm{~mm}^{3}$ ) and large plaque (volume $>271$ $\mathrm{mm}^{3}$ ). Continuous variables were categorized for worst quartiles (for clMT $>0.9 \mathrm{~mm}$, for carotid elastography SR< 0.925 and for carotid distensibility DC $\left.13.0310^{3} / \mathrm{Pa}^{-1}\right)$. Linear regression was used to estimate the association $(\beta)$ and $95 \%$ confidence interval $(\mathrm{Cl})$ between the outcomes and mean $\mathrm{BMI}$ as a 
continuous dependent variable or BMI trajectory group. Logistic regression was used to estimate the odds ratio (OR) and $95 \% \mathrm{Cl}$ for the association between the outcomes and mean BMI as a continuous dependent variable or BMI trajectory group. Primary covariates in all analyses were age and gender as they are strongly associated with both predictors and outcomes. Secondary covariates were LDL cholesterol, triglycerides, CRP, eGFR, diabetes treatment, duration of diabetes and smoking. Diabetes treatment was defined as the treatment type: no medication, oral medication, insulin, oral and insulin. For the regression models estimating the association with carotid distensibility, blood pressure was not used as a covariate since it is part of the DC equation. Statistical analysis was performed using SPSS software v24.

Calculation of trajectories- The Maccabi Diabetes Registry has BMI registered since 1998 for patients undergoing their annual visits. Trajectories of BMI were identified using a SAS macro (PROC TRAJ), which applies a multinomial modeling strategy to identify relatively homogenous clusters of developmental trajectories within a sample population. Trajectory parameters are derived by latent class analysis using maximum likelihood estimation. In particular, the distinctive trajectories of BMI were derived by modeling BMI as a function of the number of follow-up years in the Diabetes Registry prior to the start date of IDCD (defined as the intercept) with the adjustment of IDCD baseline age and gender. Distinct time points were created for each follow-up visit observed. The number of trajectories and degree of curvature were determined using the guidelines suggested by Jones et al ${ }^{19}$. For the study population mean number of BMI measurements per subject was $12.17 \pm 13.1$, with 14 subjects that had only 1 measurement of BMI. For subjects with 1 measurement, trajectory was calculated using the single measurement. Three trajectories were identified with linear, quadratic and cubic curves corresponding to normal, overweight and obese BMI groups, respectively. The output of PROC TRAJ includes the equations for the different trajectories along with the assignment of each patient to one of the trajectory groups.

\section{Results}

Subjects were $42 \%$ female, mean age of $76.4 \pm 4.4$ years, mean $\mathrm{HbA} 1 \mathrm{c}$ of $6.7 \pm 0.7 \%(50 \pm 7.7 \mathrm{mmol} / \mathrm{mol})$. Mean BMI was $29.19 \pm 4.37 \mathrm{~kg} / \mathrm{m}^{2}$, consistent with an overweight diabetic sample. Diabetes medication consumption according to medication groups at baseline was: $71 \%$ metformin, $30 \%$ sulfonylurea, $14 \%$ meglitinides, $8 \%$ insulin, $6 \%$ TZDs and 1\% GLP-1 agonists. Three types of trends of BMI over time were observed: "normal" (44\%, n=185), "overweight" (44.8\%, $\mathrm{n}=188)$, and "obese" $(11.2 \%, \mathrm{n}=47)$. The normal and overweight trajectories, represented as 1 and 2 respectively on the graph in Figure 2 , were stable over time, while the obese trajectory, represented as 3 on the graph in Figure 2, had a tendency to decline but remained stable at the obese levels.

The correlation between the different carotid atherosclerosis characteristics was estimated using the pearson $\rho$. The correlation between carotid distensibility and carotid elastography was low $\left(\rho^{2}=0.12, p=0.022\right)$. There was no correlation between cIMT with distensibility $\left(\rho^{2}=-0.01, p=0.848\right)$ or elastography

$\left(\rho^{2}=-0.043, p=0.396\right)$. Large carotid plaque volume was correlated with more clMT $\left(\rho^{2}=0.160, p=0.001\right)$ and poorer carotid elastography $\left(\rho^{2}=-0.131, p=0.007\right)$, but not with distensibility $\left(\rho^{2}=0.046, p=0.384\right)$.

Baseline characteristics by BMI trajectory groups are summarized in Table 1. BMI trajectory groups differed in age, triglycerides, HDL cholesterol, systolic and diastolic blood pressure but not in years of diabetes, mean HbA1c, LDL, eGFR, diabetes medication and smoking (Table 1). Indices of carotid stiffness and atherosclerosis by BMI trajectory groups are depicted in Figure 3. 


\begin{tabular}{|c|c|c|c|c|}
\hline & $\begin{array}{l}\text { Normal weight } \\
(n=185)\end{array}$ & $\begin{array}{l}\text { Overweight } \\
(n=188)\end{array}$ & $\begin{array}{l}\text { Obese } \\
(n=47)\end{array}$ & $\mathbf{P}$ \\
\hline Gender & & & & 0.413 \\
\hline Male & $114(61.6 \%)$ & $110(58.5 \%)$ & $24(51.1 \%)$ & \\
\hline Female & $71(38.4 \%)$ & 78 (41.5\%) & $23(48.9 \%)$ & \\
\hline Age [years] & $76.99 \pm 4.33$ & $75.95 \pm 4.41$ & $75.87 \pm 3.88$ & 0.046 \\
\hline Years diagnosed & $9.54 \pm 4.54$ & $9.71 \pm 4.49$ & $9.21 \pm 4.14$ & 0.775 \\
\hline Mean HbA1c [\%] & $6.62 \pm 0.69$ & $6.71 \pm 0.73$ & $6.75 \pm 0.8$ & 0.334 \\
\hline (mmol/mol) & $48.9 \pm 4.4$ & $49.8 \pm 5.6$ & $50.3 \pm 6.4$ & \\
\hline Triglycerides [mg/dL] & $143.45 \pm 62.61$ & $163.25 \pm 77.99$ & $171.39 \pm 62.98$ & 0.006 \\
\hline LDL cholesterol [mg/dL] & $100.98 \pm 20.72$ & $101.35 \pm 19.68$ & $97.06 \pm 20.45$ & 0.417 \\
\hline HDL cholesterol [mg/dL] & $50.21 \pm 11.57$ & $47.36 \pm 10.6$ & $45.72 \pm 9.72$ & 0.009 \\
\hline CRP $[\mu \mathrm{gr} / \mathrm{ml}]$ & $0.88 \pm 2.07$ & $1.02 \pm 1.24$ & $1.36 \pm 1.21$ & 0.208 \\
\hline Systolic BP [mmHg] & $141.42 \pm 22.12$ & $142.7 \pm 20.09$ & $153.31 \pm 25.79$ & 0.004 \\
\hline Diastolic BP [mmHg] & $70.64 \pm 9.79$ & $74.32 \pm 9.11$ & $76.26 \pm 14.38$ & 0.001 \\
\hline eGFR $\left[\mathrm{ml}^{-1} * \min ^{-1} * 1.73 \mathrm{~m}^{-2}\right]$ & & & & 0.594 \\
\hline$\leq 45$ & $5(2.7 \%)$ & $2(1.1 \%)$ & $1(2.1 \%)$ & \\
\hline $45-60$ & 29 (15.7\%) & 37 (19.9\%) & $12(25.5 \%)$ & \\
\hline$\geq 60$ & $151(81.6 \%)$ & 147 (79\%) & $34(72.3 \%)$ & \\
\hline Diabetic Medications & & & & 0.498 \\
\hline No medication & $28(56.0 \%)$ & 19 (12.3\%) & $3(0.9 \%)$ & \\
\hline Oral medication & 115 (73.7\%) & $121(78.1 \%)$ & $29(80.6 \%)$ & \\
\hline Insulin & $13(8.3 \%)$ & 15 (9.7\%) & $4(12.5 \%)$ & \\
\hline Smoking & & & & 0.269 \\
\hline Never smoked & $80(43.7 \%)$ & $65(35.5 \%)$ & $14(31.1 \%)$ & \\
\hline Smoked in the past & $85(46.4 \%)$ & $93(50.8 \%)$ & $27(60.0 \%)$ & \\
\hline Smoking & $18(9.8 \%)$ & 25 (13.7\%) & $4(8.9 \%)$ & \\
\hline
\end{tabular}

Table 1: Distribution of risk factors among the different BMI trajectory groups.

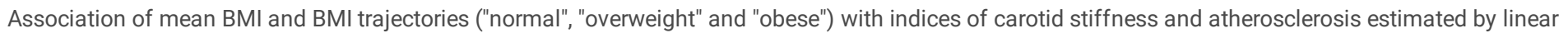

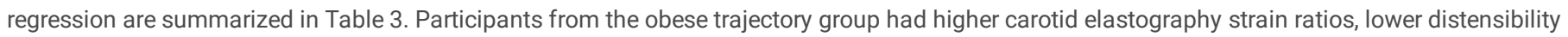
coefficients and higher cIMT than participants in the normal trajectory group.

\begin{tabular}{lccllc}
\hline & \multicolumn{2}{c}{ Normal weight } & Overweight & Obese & P \\
\hline Carotid plaque volume & $(\mathrm{n}=181)$ & $(\mathrm{n}=185)$ & $(\mathrm{n}=46)$ & 0.703 \\
& & & & \\
No plaque & $53(29.3 \%)$ & $50(27.0 \%)$ & $14(30.4 \%)$ & \\
$\quad$ Small plaque & $39(21.5 \%)$ & $49(49.0 \%)$ & $12(26.1 \%)$ & \\
$\quad$ Medium plaque & $48(26.5 \%)$ & $42(22.7 \%)$ & $7(15.2 \%)$ & \\
Large plaque & $41(22.7 \%)$ & $44(23.8 \%)$ & $13(28.3 \%)$ & \\
\hline Carotid IMT [mm] & $0.801 \pm 0.14$ & $0.819 \pm 0.16$ & $0.868 \pm 0.16$ & 0.057 \\
(n=407) & $(\mathrm{n}=166)$ & $(\mathrm{n}=169)$ & $(\mathrm{n}=36)$ & \\
\hline Carotid Distensibility [kPa & $18.39 \pm 7.46$ & $18.65 \pm 7.16$ & $15.02 \pm 6.6$ & 0.015 \\
(n=376) & $(\mathrm{n}=154)$ & $(\mathrm{n}=154)$ & $(\mathrm{n}=41)$ & \\
\hline Carotid Elastography [SR] & $0.757 \pm 0.26$ & $0.757 \pm 0.31$ & $0.932 \pm 0.46$ & 0.003 \\
(n=432) & $(\mathrm{n}=178)$ & $(\mathrm{n}=179)$ & $(\mathrm{n}=42)$ & \\
\hline
\end{tabular}

Table 2: Distribution of atherosclerosis markers between the BMI trajectory groups. 


\begin{tabular}{llccc}
\hline Dependent variable & & $\beta$ & CI & p-value \\
\hline Distensibility coefficient* & Mean BMI & -0.275 & {$[-0.469,-0.082]$} & 0.005 \\
& Normal weight & 0 & & \\
& Overweight & 0.170 & {$[-1.584,1.924]$} & 0.849 \\
& Obese & -3.078 & {$[-5.974,-0.182]$} & 0.037 \\
\hline Elastography strain ratio & Mean BMI & 0.006 & {$[-0.002,0.015]$} & 0.139 \\
\cline { 2 - 5 } & Normal weight & 0 & & \\
& Overweight & -0.014 & {$[-0.086,0.057]$} & 0.698 \\
& Obese & 0.181 & {$[0.058,0.304]$} & 0.004 \\
\hline CIMT & Mean BMI & 0.005 & {$[0.001,0.009]$} & 0.026 \\
\hline & Normal weight & 0 & & \\
& Overweight & 0.033 & {$[-0.002,0.069]$} & 0.068 \\
& Obese & 0.095 & {$[0.030,0.160]$} & 0.004 \\
\hline Plaque volume group & \\
& Mean BMI & -0.005 & {$[-0.053,0.044]$} & 0.843 \\
& Normal weight & CG & & \\
& Overweight & 0.146 & {$[-0.286,0.577]$} & 0.508 \\
& Obese & 0.066 & {$[-0.661,0.793]$} & 0.858 \\
\hline
\end{tabular}

Table 3: Association of mean BMI and BMI trajectories ("normal", "overweight" and "obese") with indices of carotid stiffness and atherosclerosis estimated by linear regression. Models were adjusted to age, gender, diabetes parameters (years of diabetes, medication and HbA1c), blood lipids, CRP, blood pressure, eGFR and smoking status.

* Distensibility was not adjusted to blood pressure; $†$ Plaque volume association was estimated by ordinal regression for carotid plaque volume group.

Association of mean BMI and BMI trajectories with cutoffs for indices of carotid stiffness and atherosclerosis estimated by logistic regressions are summarized in Table 4. Participants from the obese trajectory group had a 2.79-fold increased odds for impaired distensibility, 5.28-fold increased odds for thickened cIMT and 8.46-fold increased odds for large plaque volume compared with the normal BMI trajectory group.

\begin{tabular}{llcccr}
\hline Dependent variable & & OR & CI & p-value \\
\hline Distensibility coefficient & Mean BMI & 1.080 & {$[1.011,1.153]$} & 0.022 \\
& Normal weight & CG & & \\
& Overweight & 1.083 & {$[0.565,2.047]$} & 0.810 \\
& Obese & 2.790 & {$[1.087,7.158]$} & 0.033 \\
\hline Elastography strain ratio & Mean BMI & 1.035 & {$[0.972,1.103]$} & 0.278 \\
& Normal weight & CG & & \\
& Overweight & 1.138 & {$[0.630,2.065]$} & 0.668 \\
& Obese & 1.965 & {$[0.801,4.818]$} & 0.140 \\
\hline CIMT & Mean BMI & 1.126 & {$[1.051,1.206]$} & 0.001 \\
\hline Normal weight & CG & & \\
& Overweight & 2.294 & {$[1.264,4.164]$} & 0.006 \\
& Obese & 5.277 & {$[2.013,13.838]$} & 0.001 \\
\hline Carotid plaque volume & Mean BMI & 0.995 & {$[0.941,1.052]$} & 0.866 \\
& Normal weight & CG & & \\
& Overweight & 2.205 & {$[0.967,5.027]$} & 0.060 \\
& Obese & 8.456 & {$[1.559,45.863]$} & 0.013 \\
\hline
\end{tabular}

Table 4: Risk ratio estimation of mean BMI and BMI trajectories ("normal", "overweight" and "obese") with indices of carotid stiffness and atherosclerosis estimated by logistic regression. Models were adjusted to age, gender, diabetes parameters (years of diabetes, medication and HbA1c), CRP, blood lipids, blood pressure, eGFR and smoking status.

* Distensibility was not adjusted to blood pressure.

An additional sub-study $(n=36)$ for the repeatability and reliability of carotid plaque volume measurement was conducted, demonstrating good intra-observer $(I C C=0.92, p<0.001)$ and inter-observer $(I C C=0.90, p<0.001)$ agreement. For post processing of plaque volume there was excellent intra-observer (ICC=0.97, $\mathrm{p}<0.001)$ and inter-observer $(I C C=0.84, p<0.001)$ agreement.

\section{Discussion}

In this study we have found that among elderly cognitively normal patients with type 2 diabetes, that longitudinal measurements of adiposity are associated with indices of carotid stiffness and atherosclerotic plaque volume. Adjustment of the models for several relevant cardiovascular and sociodemographic 
variables did not attenuate these associations.

This study provides new evidence in several levels. The study focuses on an elderly diabetic population on which there is scarce evidence on relationships of obesity with measures of carotid stiffness and atherosclerotic plaque volume, despite the biological plausibility of this association. Using a broad battery, we have found that adiposity is associated differentially with carotid indices, suggesting that adiposity affects some carotid features more than others. Finally, we have used long-term data of BMI that span approximately 26 years and presented both as mean of all measurements and trajectories of BMI.

In this study we have found that BMI is associated with carotid artery stiffening as indicated by carotid distensibility and elastography, in addition to the association with carotid atherosclerosis parameters as indicated by cIMT and carotid plaque volume. Arteries are known to stiffen in healthy aging and with atherosclerosis, diabetes, hypertension and obesity ${ }^{20}$. Stiffening of the carotid arteries is associated with higher risk for stroke ${ }^{21}$ and is predictive of white matter hyperintensity volume and total brain volume ${ }^{22}$. It has been suggested that stiffening of the carotid artery increases the mechanical force on existing plaque and as a result increases the risk for rupture of existing plaques ${ }^{23}$.

Our data provide additional support to the growing evidence on the association of obesity with impaired vascular health. Impaired vascular health in the carotid artery wall was observed as early as in hypertensive children ${ }^{24}$, healthy pre- and early pubescent children ${ }^{25}$, adolescents ${ }^{9,26}$, and physically inactive adults office workers ${ }^{6}$. In a case control study among adults, obesity was associated with cIMT (but not distensibility) ${ }^{7,27}$. Increased cIMT and decreased distensibility were observed among adult men ${ }^{28}$ and women ${ }^{29,30}$. The long-term effects of increased childhood BMI on adulthood carotid atherosclerosis was examined in several studies. In two cohort studies BMI trajectories that were identified from childhood to adulthood increased the risk for impaired clMT ${ }^{31,32}$. An additional study found an association of BMI in childhood, young adulthood and cumulative risk with impaired clMT among healthy population ${ }^{33}$. In addition, obesity was found to be the most influencing risk factor for unstable carotid plaque among men under $70^{34}$. A research looking at CVD risk trajectories from childhood to adulthood found that many of those who had consistently high BMls during the life-course were better protected from carotid intima-media thickening if they were physically active ${ }^{35}$. Furthermore, in additional studies, no association was found between BMI and carotid atherosclerosis in non-diabetic ${ }^{36}$ as well as diabetic patients ${ }^{5,37}$. We are not aware of any published evidence on the association of carotid elastography and BMI.

An additional aspect is variability in BMI over time, which has been associated with other old age outcomes such as dementia ${ }^{46}$. BMI variability over time in addition to the trajectory of BMI over time, might affect carotid atherosclerosis. We have examined the associations of standard deviation of all BMI measures available for each subject with the carotid measures and found no significant associations (data not shown) suggesting that in this sample of older adults, variability in BMI does not predict carotid vascular health.

Several underlying biological mechanisms may link BMI with impaired vascular health. In our study obese patients were younger, yet had higher triglycerides and blood pressure, and lower HDL cholesterol, all independent risk factors for atherosclerosis in general and carotid artery atherosclerosis in particular ${ }^{38}$. However, adjusting for these risk factors did not attenuate the BMI-carotid associations suggesting involvement of other mechanisms. Second, adipocytokines, i.e. fat-related inflammatory markers such as IL-6 and leptin, play an important role in atherosclerosis including initial activation of endothelial cells, through atherosclerotic progression and, ultimately, its final complication, thrombosis ${ }^{39}$. As was shown in a cohort of elderly patients with type 2 diabetes, adipose tissue-derived inflammatory factors resistin, vaspin and visfatin were associated with pathogenesis of carotid atherosclerosis ${ }^{40}$. Third, visceral abdominal fat has a direct circulatory connection to the liver. Excessive release of free fatty acids from visceral adipose tissue directly in the portal circulation might lead to insulin resistance and hyperlipidemia, both established risk factors for CVD ${ }^{41}$.

Our study has several limitations. The study is conducted on elderly non-demented type 2 diabetes patients which may reflect a population of "survivors", so subjects who were eligible for this study were those who were not demented after 2 IDCD follow up visits over approximately four years. The IDCD study focuses on older adults with type 2 diabetes and thus conclusions from this study cannot be extrapolated to non-diabetic populations. We have only crosssectional carotid artery data so reverse causality cannot be ruled out. However, the BMI trajectories spanned 26 years suggesting that long-term obesity may be a predictor of carotid disease.

An additional limitation is that our measure of adiposity was BMI, so we could not directly address aspects of body composition such as visceral fat or fat distribution ${ }^{42,43,44}$. There is, however, evidence that in children combination of total and central measures of fat does not improve the prediction of increased cIMT, and a simple surrogate measures of fatness such as BMI can be used ${ }^{45}$.

Strengths of our study include its relatively large-scale, the in depth carotid assessments including novel indices of impaired vascular health, a directly measured (rather than self-reported) diabetes diagnosis, and an exquisite characterization of long-term covariates and of BMI trajectories derived from the Maccabi Diabetes Registry data.

\section{Conclusion}

Our results suggest that mean BMI and BMI trajectories of obesity are associated with subclinical atherosclerosis and impaired vascular health among elderly non demented type 2 diabetes patients. Further studies should assess the effect of weight reduction on subclinical carotid atherosclerosis, and whether the associations of BMI with macro-vascular complications of diabetes such as stroke are mediated by carotid atherosclerosis.

\section{Abbreviations}

BMI - body mass index 
CCA - common carotid artery

$\mathrm{Cl}$ - confidence interval

cIMT - carotid Intima Media Thickness

$\mathrm{HMO}$ - health maintenance organization

IDCD - Israel Diabetes and Cognitive Decline

MHS - Maccabi Healthcare Services

OR - odds ratio

SR - strain ration

VPQ - Vascular Plaque Quantification

$\mathrm{ROI}$ - region of interest

\section{Declarations}

\section{Acknowledgments}

Acknowledgement is made to the donors of Alzheimer's Disease Research, a program of BrightFocus Foundation, for support of this research and NIH grants AG053446, AG051545, AG034087 and AG043878.

Ethics approval and consent to participate - The study was approved by the Institutional Review Board of Mount Sinai and the Helsinki committees of Sheba and MHS.

Consent for publication - not applicable

Availability of data and materials - The datasets used and/or analysed during the current study are available from the corresponding author on reasonable request.

Competing interests - The authors declare that they have no competing interests

Funding - This study was funded by grants from NIH grants AG053446, AG051545, AG034087 and AG043878 and donors of Alzheimer's Disease Research, a program of BrightFocus Foundation.

\section{Authors contribution -}

CBM - data collection, analysis and interpretation of data, drafted the work

$\mathrm{SH}$ - design of the work, interpretation of data

RRS - design of the work

$\mathrm{AH}$ - substantial contribution to the conception

MSB - substantial contributions to the conception, design of the work, interpretation of data, substantively revised the work

DT - substantial contributions to the conception, design of the work, interpretation of data, substantively revised the work

Acknowledgements - to the donors of Alzheimer's Disease Research, a program of BrightFocus Foundation, for support of this research and NIH grants AG053446, AG051545, AG034087 and AG043878.

\section{References}

1. Adela Hruby, PhD M, Frank B. Hu, MD, PhD M. The Epidemiology of Obesity: A Big Picture. Pharmacoeconomics. 2015;33(7):673-689. doi:10.1007/s40273-014-0243-x.The

2. Inoue Y, Qin B, Poti J, Sokol R, Gordon-Larsen P. Epidemiology of Obesity in Adults: Latest Trends. Curr Obes Rep. 2018;7(4):276-288. doi:10.1007/s13679018-0317-8

3. Smith KB, Smith MS. Obesity Statistics. Prim Care - Clin Off Pract. 2016;43(1):121-135. doi:10.1016/j.pop.2015.10.001

4. Amer MS, Khater MS, Omar OH, Mabrouk R a, Mostafa S a. Association between Framingham risk score and subclinical atherosclerosis among elderly with both type 2 diabetes mellitus and healthy subjects. Am J Cardiovasc Dis. 2014;4(1):14-19.

5. Jung $\mathrm{CH}, \mathrm{Kim} \mathrm{BY}, \mathrm{Kim} \mathrm{KJ}$, et al. Contribution of subcutaneous abdominal fat on ultrasonography to carotid atherosclerosis in patients with type 2 diabetes mellitus. Cardiovasc Diabetol. 2014;13(1):1-8. doi:10.1186/1475-2840-13-67

Page $8 / 12$ 
6. Jin Y, Kim D, Cho J, Lee I, Choi K, Kang H. Association between Obesity and Carotid Intima-Media Thickness in Korean Office Workers: The Mediating Effect of Physical Activity. Biomed Res Int. 2018;2018. doi:10.1155/2018/4285038

7. Prado SS, Ribeiro ML, Cardoso GP, Bousquet-Santos K, Da Nóbrega ACL, Velarde LGC. Carotid artery structural and functional evaluation in relatives of type 2 diabetic patients. Arq Bras Cardiol. 2009;92(3):190-196. doi:10.1590/S0066-782X2009000300006

8. Geerts CC, Evelein AMV, Bots ML, Van Der Ent CK, Grobbee DE, Uiterwaal CSPM. Body fat distribution and early arterial changes in healthy 5-year-old children. Ann Med. 2012;44(4):350-359. doi:10.3109/07853890.2011.558520

9. Jourdan C, Wühl E, Litwin M, et al. Normative values for intima-media thickness and distensibility of large arteries in healthy adolescents. $J$ Hypertens. 2005;23(9):1707-1715. doi:10.1097/01.hjh.0000178834.26353.d5

10. Simon A, Megnien JL, Chironi G. The value of carotid intima-media thickness for predicting cardiovascular risk. Arterioscler Thromb Vasc Biol. 2010;30(2):182-185. doi:10.1161/ATVBAHA.109.196980

11. Gardin JM, Bartz TM, Polak JF, Daniel H, Leary O, Wong ND. Prediction of Stroke and Cardiovascular Disease Risk in Older. 2015;27(November 2012):9981005. doi:10.1016/j.echo.2014.06.013. What

12. Roumeliotis A, Roumeliotis S, Panagoutsos S, et al. Carotid intima-media thickness is an independent predictor of all-cause mortality and cardiovascular morbidity in patients with diabetes mellitus type 2 and chronic kidney disease. Ren Fail. 2019;41(1):131-138. doi:10.1080/0886022X.2019.1585372

13. Kawasaki T, Sasayama S, Yagi SI, Asakawa T, Hirai T. Non-invasive assessment of the age related changes in stiffness of major branches of the human arteries. Cardiovasc Res. 1987;21(9):678-687. doi:10.1093/cvr/21.9.678

14. Van Popele NM, Grobbee DE, Bots ML, et al. Association between arterial stiffness and atherosclerosis: The Rotterdam study. Stroke. 2001;32(2):454-460. doi:10.1161/01.STR.32.2.454

15. Beeri MS, Ravona-Springer R, Moshier E, et al. The Israel Diabetes and Cognitive Decline (IDCD) study: Design and baseline characteristics. Alzheimer's Dement. 2014;10(6):769-778. doi:10.1016/j.jalz.2014.06.002

16. Alexandrov A V., Sloan MA, Wong LKS, et al. Practice standards for transcranial Doppler ultrasound: Part I - Test performance. J Neuroimaging. 2007;17(1):11-18. doi:10.1111/j.1552-6569.2006.00088.x

17. PJ Touboul, MG Hennerici, S Meairs, H Adams, P Amarenco, N Bornstein, L Csiba M, Desvarieux, S Ebrahim, R. Hernandez Hernandez, M Jaff, S Kownator, T Naqvi, P Prati T, Rundek, M Sitzer, U Schminke, JC Tardif, A Taylor, E Vicaut and KW. Mannheim Carotid Intima-Media Thickness and Plaque Consensus (2004-2006-2011): An Update. Cerebrovasc Dis. 2012;34(4):290-296. doi:10.1159/000343145.Mannheim

18. Ravona-Springer R, Heymann A, Schmeidler J, et al. Trajectories in Glycemic Control over Time Are Associated with Cognitive Performance in Elderly Subjects with Type 2 Diabetes. PLoS One. 2014;9(6):e97384. doi:10.1371/journal.pone.0097384

19. Jones BL, Nagin DS, Roeder K. A SAS procedure based on mixture models for estimating developmental trajectories. Sociol Methods Res. 2001;29(3):374393. doi:10.1177/0049124101029003005

20. Boesen ME, Singh D, Menon BK, Frayne R. A systematic literature review of the effect of carotid atherosclerosis on local vessel stiffness and elasticity. Atherosclerosis. 2015;243(1):211-222. doi:10.1016/j.atherosclerosis.2015.09.008

21. Yang EY, Chambless $L$, Sharrett AR, et al. Carotid arterial wall characteristics are associated with incident ischemic stroke but not coronary heart disease in the Atherosclerosis Risk in Communities (ARIC) Study. Stroke. 2012;43(1):103-108. doi:10.1161/STROKEAHA.111.626200

22. de Havenon A, Wong K-H, Elkhetali A, McNally JS, Majersik JJ, Rost NS. Carotid Artery Stiffness Accurately Predicts White Matter Hyperintensity Volume 20 Years Later: A Secondary Analysis of the Atherosclerosis Risk in the Community Study. Am J Neuroradiol. 2019;40(8):1369 LP - 1373. doi:10.3174/ajnr.A6115

23. Arroyo LH, Lee RT. Mechanisms of plaque rupture: Mechanical and biologic interactions. Cardiovasc Res. 1999;41(2):369-375. doi:10.1016/S00086363(98)00308-3

24. Litwin M, Trelewicz J, Wawer Z, et al. Intima-media thickness and arterial elasticity in hypertensive children: Controlled study. Pediatr Nephrol. 2004;19(7):767-774. doi:10.1007/s00467-004-1480-6

25. Banach AM, Peralta-Huertas J, Livingstone K, et al. Arterial distensibility is reduced in overweight pre- and early pubescent children. Eur J Pediatr. 2010;169(6):695-703. doi:10.1007/s00431-009-1092-z

26. Kozakova M, Morizzo C, Bianchi V, Marchetti S, Federico G, Palombo C. Hemodynamic overload and intra-abdominal adiposity in obese children: Relationships with cardiovascular structure and function. Nutr Metab Cardiovasc Dis. 2016;26(1):60-66. doi:10.1016/j.numecd.2015.10.002

27. Purnamasari D, Abdaly MS, Azizi MS, Wijaya IP, Nugroho P. $<\mathrm{p}>$ Carotid intima-media thickness among normoglycemia and normotension first-degree relatives of type 2 diabetes mellitus</p>. Vasc Health Risk Manag. 2019;Volume 15:101-107. doi:10.2147/vhrm.s195776

28. Moore XL, Michell D, Lee S, et al. Increased Carotid Intima-Media Thickness and Reduced Distensibility in Human Class III Obesity: Independent and Differential Influences of Adiposity and Blood Pressure on the Vasculature. PLoS One. 2013;8(1). doi:10.1371/journal.pone.0053972

29. P.E. Z, T. N, L. T, et al. Obesity is associated with increased arterial stiffness from adolescence until old age. J Hypertens. 2005;23(10):1839-1846. doi:10.1097/01.hjh.0000179511.93889.e9 LK - http://sfx.library.uu.nl/utrecht?

sid=EMBASE\&issn=02636352\&id=doi:10.1097\%2F01.hjh.0000179511.93889.e9\&atitle=Obesity+is+associated+with+increased+arterial+stiffness+from+c 1846\&date $=2005$ \&auinit1 $=P \&$ auinitm $=E$

30. Ren C, Zhang J, Xu Y, et al. Association between carotid intima-media thickness and index of central fat distribution in middle-aged and elderly Chinese. Cardiovasc Diabetol. 2014;13(1):1-8. doi:10.1186/s12933-014-0139-2

31. Buscot MJ, Thomson RJ, Juonala M, et al. Distinct child-to-adult body mass index trajectories are associated with different levels of adult cardiometabolic risk. Eur Heart J. 2018;39(24):2263-2270a. doi:10.1093/eurheartj/ehy161

Page $9 / 12$ 
32. Hao G, Wang X, Treiber FA, Harshfield G, Kapuku G, Su S. Body mass index trajectories in childhood is predictive of cardiovascular risk: results from the 23-year longitudinal Georgia Stress and Heart study. Int J Obes. 2018;42(4):923-925. doi:10.1038/ijo.2017.244

33. Bogalusa T, Study H, Bond MG, Urbina EM, Berenson GS. Childhood Cardiovascular Risk Factors. Lancet. 2003;290(17):2271-2276.

34. Rovella V, Anemona L, Cardellini M, et al. The role of obesity in carotid plaque instability: Interaction with age, gender, and cardiovascular risk factors. Cardiovasc Diabetol. 2018;17(1):1-9. doi:10.1186/s12933-018-0685-0

35. Pollock BD, Stuchlik P, Harville EW, et al. Life course trajectories of cardiovascular risk: Impact on atherosclerotic and metabolic indicators. Atherosclerosis. 2019;280(August 2018):21-27. doi:10.1016/j.atherosclerosis.2018.11.008

36. De Barros IL, Costa L, Bezerra B, et al. Predictors of subclinical carotid atherosclerosis in middle-aged women. PLoS One. 2018;13(5):1-10. doi:10.1371/journal.pone.0197582

37. Kupfer R, Larrúbia MR, Bussade I, et al. Predictors of subclinical atherosclerosis evaluated by carotid intima-media thickness in asymptomatic young women with type 1 diabetes mellitus. Arch Endocrinol Metab. 2017;61(2):115-121. doi:10.1590/2359-3997000000255

38. Silaghi CA, Silaghi H, Crăciun AE, et al. Age, abdominal obesity, and glycated hemoglobin are associated with carotid atherosclerosis in type 2 diabetes patients with nonalcoholic fatty liver disease. Med Ultrason. 2015;17(3):300-307. doi:10.11152/mu.2013.2066.173.cas

39. Ammirati E, Moroni F, Norata GD, Magnoni M, Camici PG. Markers of inflammation associated with plaque progression and instability in patients with carotid atherosclerosis. Mediators Inflamm. 2015;2015. doi:10.1155/2015/718329

40. Yang W, Li Y, Wang JY, Han R, Wang L. Circulating levels of adipose tissue-derived inflammatory factors in elderly diabetes patients with carotid atherosclerosis: A retrospective study. Cardiovasc Diabetol. 2018;17(1):1-9. doi:10.1186/s12933-018-0723-y

41. Han TS, Lean ME. A clinical perspective of obesity, metabolic syndrome and cardiovascular disease. JRSM CardiovasC Dis. 2016;5:204800401663337. doi:10.1177/2048004016633371

42. Ashwell M, Gunn P, Gibson S. Waist-to-height ratio is a better screening tool than waist circumference and BMI for adult cardiometabolic risk factors: Systematic review and meta-analysis. Obes Rev. 2012;13(3):275-286. doi:10.1111/j.1467-789X.2011.00952.x

43. Adegbija O, Hoy WE, Wang Z. Waist circumference values equivalent to body mass index points for predicting absolute cardiovascular disease risks among adults in an Aboriginal community: A prospective cohort study. BMJ Open. 2015;5(11):1-7. doi:10.1136/bmjopen-2015-009185

44. De Koning L, Merchant AT, Pogue J, Anand SS. Waist circumference and waist-to-hip ratio as predictors of cardiovascular events: Meta-regression analysis of prospective studies. Eur Heart J. 2007;28(7):850-856. doi:10.1093/eurheartj/ehm026

45. Melo X, Santa-Clara H, Santos DA, et al. Single and combined effects of body composition phenotypes on carotid intima-media thickness. Pediatr Obes. 2016;11(4):272-278. doi:10.1111/ijpo.12056

46. Ravona-Springer R, Schnaider-Beeri M, Goldbourt U. Body weight variability in midlife and risk for dementia in old age. Neurology. 2013;80(18):1677-1683. doi:10.1212/WNL.0b013e3182904cee

\section{Figures}

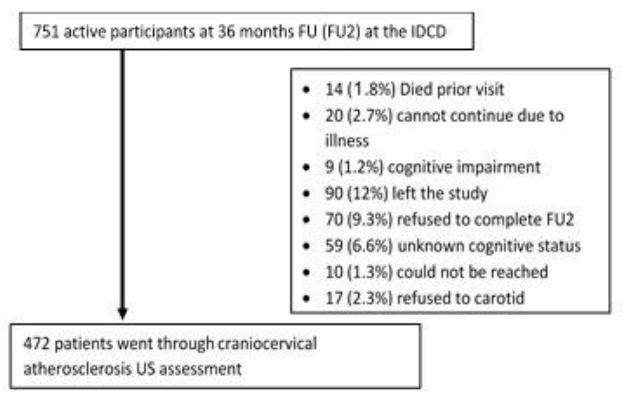

Figure 1: Flow chart of the patients in the IDCD carotid artery cohort

\section{Figure 1}

Flow chart of the patients in the IDCD carotid artery cohort 


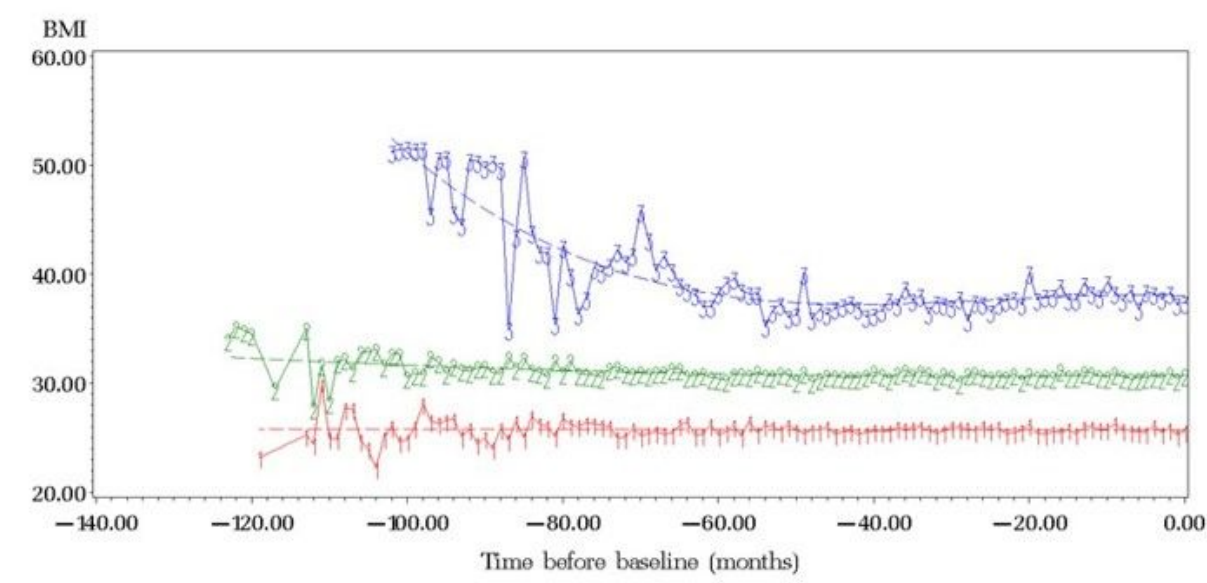

Figure 2: BMI groups. Three types of trends of BMI over time: 1-"normal", 2-"overweight" and 3-"obese"

Figure 2

BMI groups. Three types of trends of BMI over time: 1-"normal", 2-"overweight" and 3-"obese"
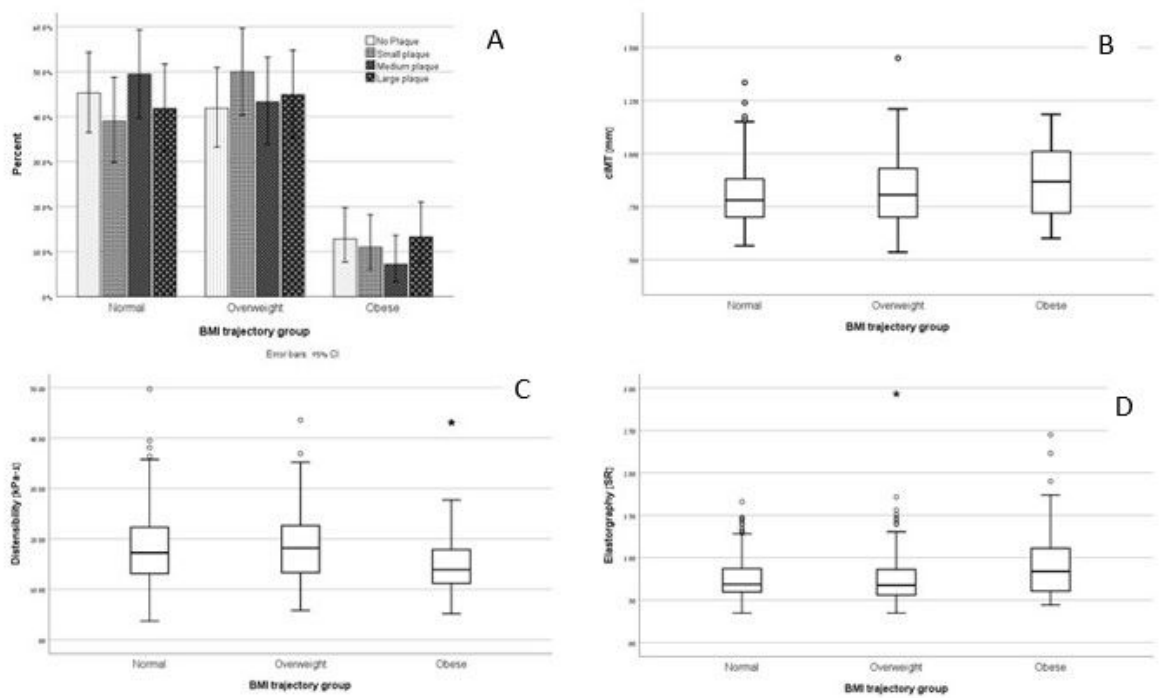

Figure 3: Distribution of the carotid atherosclerosis measurements among the 3 trajectory groups: (A) plaque volume prevalence $(B)$ clML (C) Distensibility and $(D)$ eldastegreaphlu.

\section{Figure 3}

Distribution of the carotid atherosclerosis measurements among the 3 trajectory groups: (A) plaque volume prevalence (B) clMT (C) Distensibility and (D) elastogreaphy.

\section{Supplementary Files}

This is a list of supplementary files associated with this preprint. Click to download. 
- onlinesupplementary.pdf

Page 12/12 